\title{
Transrectus sheath pre-peritoneal (TREPP) procedure versus totally extraperitoneal (TEP) procedure and Lichtenstein technique: a propensity-score-matched analysis in Dutch high-volume regional hospitals
}

\author{
T. L. R. Zwols ${ }^{1,8}$ (1) N. Slagter ${ }^{1}$ - N. J. G. M. Veeger ${ }^{2} \cdot$ M. J. W. Möllers ${ }^{3} \cdot$ D. A. Hess ${ }^{4} \cdot$ E. Jutte ${ }^{1} \cdot$ H. T. Brandsma ${ }^{4}$ \\ P. H. J. M. Veldman ${ }^{5}$. G. G. Koning ${ }^{6}$ H. H. Eker ${ }^{1}$. J. P. E. N. Pierie ${ }^{1,7,8}$
}

Received: 19 May 2020 / Accepted: 24 August 2020 / Published online: 16 October 2020

(c) The Author(s) 2020

\begin{abstract}
Purpose Results of the most commonly used inguinal hernia repair techniques often originate from expert centers or from randomized controlled studies. In this study, we portray daily-practice results of a high-volume, regional surgical group in the Netherlands, comparing TREPP (open (posterior) transrectus sheath pre-peritoneal) with Lichtenstein (open anterior) and TEP (endoscopic (posterior) totally extraperitoneal). We hypothesize that the TREPP shows more favorable outcome compared to the current gold standard procedures: TEP and Lichtenstein.

Methods Between January 2016 and December 2018, 3285 consecutive patients underwent surgical treatment and were included for analysis. The outcome measures were postoperative pain, recurrence rate and other surgical complications. Propensity-score matching was used to address potential selection bias.

Results After propensity-score matching, there was no statistically significant difference in postoperative pain in the TREPP group compared to the Lichtenstein group (TREPP 7.3\% versus Lichtenstein 6.3\%; $p=0.67$ ) nor in TREPP compared to TEP (TREPP $7.4 \%$ versus TEP $4.1 \% ; p=0.064$ ). There was no statistically significant difference in recurrences in the TREPP group compared to Lichtenstein ( $3.8 \%$ vs $2.5 \%$; $p=0.42$ ), nor in the TREPP versus TEP comparison $(3.9 \%$ vs $2.8 \% ; p=0.55)$ Conclusion This study compares TREPP with Lichtenstein and TEP in the presence of postoperative pain, recurrences and other adverse outcomes. After propensity-score matching, no statistically significant difference in postoperative pain or recurrences remained between either TREPP compared to Lichtenstein, or TREPP compared to TEP. Based on these results, TREPP, Lichtenstein and TEP showed comparable results in postoperative pain, recurrences and other surgical site complications.
\end{abstract}

Keywords TEP $\cdot$ Lichtenstein $\cdot$ TREPP $\cdot$ Open preperitoneal $\cdot$ Inguinal hernia repair · Groin hernia

T. L. R. Zwols

thomas.zwols@znb.nl

1 Department of Surgery, Medisch Centrum Leeuwarden, Leeuwarden, The Netherlands

2 Department of Clinical Epidemiology, Medisch Centrum Leeuwarden, Leeuwarden, The Netherlands

3 Department of Surgery, Nij Smellinghe, Drachten, The Netherlands

4 Department of Surgery, Antonius Ziekenhuis, Sneek, The Netherlands
5 Department of Surgery, Tjongerschans Heerenveen, Heerenveen, The Netherlands

6 Department of Surgery, Ikazia Ziekenhuis, Rotterdam, The Netherlands

7 Postgraduate School of Medicine, University Medical Centre Groningen, Groningen, The Netherlands

8 The University Medical Centre Groningen, University of Groningen, Groningen, The Netherlands 


\section{Introduction}

Inguinal hernia repair is one of the most frequently performed surgical procedures worldwide with up to 20 million patients annually [1]. In the Netherlands, approximately 30,000 patients undergo inguinal hernia repair each year [2]. The current recommendation for patients who experience symptoms of inguinal hernia such as pain or mechanical complaints is elective inguinal hernia repair. International guidelines recommend conservative treatment without surgery if the patient has no complaints. Studies have shown, however, that approximately $70 \%$ of patients will have an increase in complaints and will eventually require inguinal hernia repair $[3,4]$.

In the current guidelines, the preferred surgical technique in any elective inguinal hernia repair depends on many different characteristics and can differ between patients. In general, a surgical technique that includes mesh to strengthen the abdominal wall is recommended [1]. The totally extraperitoneal (TEP) procedure is the recommended endoscopic technique for elective inguinal hernia repair. The Lichtenstein technique is recommended for patients who are not fit for general anesthesia, in case of recurrence or when minimal invasive equipment or experience is lacking. In patients where mesh placement is impossible or where circumstances make mesh placement less opportune, the Shouldice technique is the recommended non-mesh technique for inguinal hernia repair [1].

TEP and Lichtenstein show similar perioperative complications and operating times [5]. After the introduction of mesh placement in elective inguinal hernia repair, the rate of recurrences dropped. With this lower rate of recurrences, another outcome of inguinal hernia repair became most prevalent: chronic postoperative inguinal pain (CPIP), which is defined as any inguinal pain $>3$ months after surgery [6]. The overall incidence of CPIP is approximately $11 \%$ [7]. The prevalence of patients that are affected with CPIP in daily activities or work ranges from 0.5 to $6 \%$ [1]. The TEP shows slightly better results in the development of chronic postoperative inguinal pain (CPIP) and return to normal daily activities in comparison to the Lichtenstein technique [5, 8].

For the Lichtenstein technique, prevalence varies up to $29 \%$ [5, 8-10]. After TEP, these percentages vary up to $12 \%[5,8,9]$. For the transrectus sheath pre-peritoneal (TREPP) procedure, there is less literature available, but the existing literature suggests a lower percentage of pain around 5\% [11].

Most results originate from high-volume expert centers where one specific technique is used or from randomized studies that usually include a specific subset of the general population. The cumulative results of these specific studies have become the textbook-outcome results but may differ from the general daily practice. In this study, we portray daily-practice results of inguinal hernia surgery performed in four high-volume regional hospitals in the Netherlands, both teaching and non-teaching, on TREPP, TEP and Lichtenstein. The aim of this study is to evaluate textbook-outcome results on inguinal hernia surgery in the daily practice in regional hospitals, to provide both doctors and patients with more insights on the risk of complications after inguinal hernia surgery. We hypothesize that TREPP results in a more favorable outcome (postoperative pain, recurrence) compared to the gold standard procedures TEP and Lichtenstein.

\section{Patients and methods}

\section{Study design}

The Medical Ethics Committee of our institution (RTPO Leeuwarden, The Netherlands) confirmed that this retrospective study could be carried out without the need for ethical review, and the institutional boards of the four hospitals approved the execution of the study without the need for consent in accordance with Dutch regulations. All participating centers provided data of experienced hernia surgeons (at least 100 inguinal hernia procedures performed) performing or supervising the surgical treatment. The aim of this study was to evaluate the daily practice in inguinal hernia surgery in four teaching and non-teaching hospitals in the northern region of the Netherlands. The primary outcome of the study was to evaluate the development of postoperative pain. The secondary outcomes are surgical site occurrences (recurrence, haematoma, seroma, bleeding, wound infection, abscess, urinary tract infection, ileus).

\section{Retrospective database}

All patients with a primary or recurrent, direct or indirect, inguinal or femoral hernia, in an acute or elective setting were included in the database. Patients who did not undergo surgical treatment were excluded from the database. Patients were operated with different anterior or posterior techniques, e.g., Lichtenstein, TEP, TAPP, TREPP, TIPP, Bassini, Shouldice, Stoppa, Rutkow-Robbins, Fabricius. The choice of technique was dependent on the surgeon and on whether or not it concerned a primary or recurrent hernia. For this study, analyses were performed on all adult male patients with primary, unilateral inguinal hernia, who were treated in the elective setting. 


\section{Outcome measures}

Patients were scheduled for regular follow-ups at the outpatient clinic at two-six weeks postoperatively. More visits were scheduled only in case of adverse events. Every outcome that was mentioned in the electronic patient file was noted in the database. For postoperative pain specifically, patients scored a "yes" if they: visited the outpatient clinic after a regular follow-up because of inguinal pain; received pain treatment or had any further pain evaluation (e.g., ultrasonography, MR-imaging, referral to pain specialist).

\section{Statistical analysis}

To address potential selection bias due to the observational nonrandomized study design, we implemented propensityscore matching to achieve a more balanced study cohort. In this, patients treated with TREPP were 1:1 matched to patients treated with Lichtenstein, using their probability to receive TREPP, i.e., the propensity score for TREPP. Matching was performed with a 0.01 maximum allowed difference in the exact propensity scores in a 'pair of patients' treated with TREPP and Lichtenstein. Propensity score of individual patients was estimated using multivariable logistic regression with covariates describing condition at baseline. In addition, patients treated with TREPP were matched to TEP used the same procedure.

Subsequent analyses were performed using the matched cohorts. Percentages of outcome were calculated and exact odds ratios with $95 \%$ confidence intervals estimated. For this, exact conditional logistic regression was used to control for potential lack of independence due to matched pairs.

Baseline characteristics between groups were analyzed using Chi-square test and a two-tailed Fisher exact test. Outcome measures in both cohorts were analyzed using a two-tailed Fisher exact test. $p$ values less than 0.05 were considered statistically significant. Statistical analysis was performed with SAS 9.2 (SAS Institute Inc. Cary, NY, USA).

\section{Results}

\section{Patient characteristics}

In the period between January 1st, 2016 and December 31st, 2018, a total of 3762 inguinal hernia repairs were performed. Surgeries were performed in four regional hospitals, one teaching and three non-teaching. The flowchart of selected patients for analysis in this study is shown in Fig. 1.

\section{Baseline characteristics}

Before propensity-score matching (PSM), the cohort undergoing the TREPP procedure $(n=398)$ was younger (mean age $57.0 ; 18-88$ versus 63.7 years; $18-95$ ) than the Lichtenstein cohort $(n=1051)$. The TEP cohort $(n=552)$ had the lowest mean age (54.3 years; $18-88$ ). Baseline patient characteristics are shown in Table 1.
Fig. 1 Flowchart of total inguinal hernia repairs within the selected timeframe

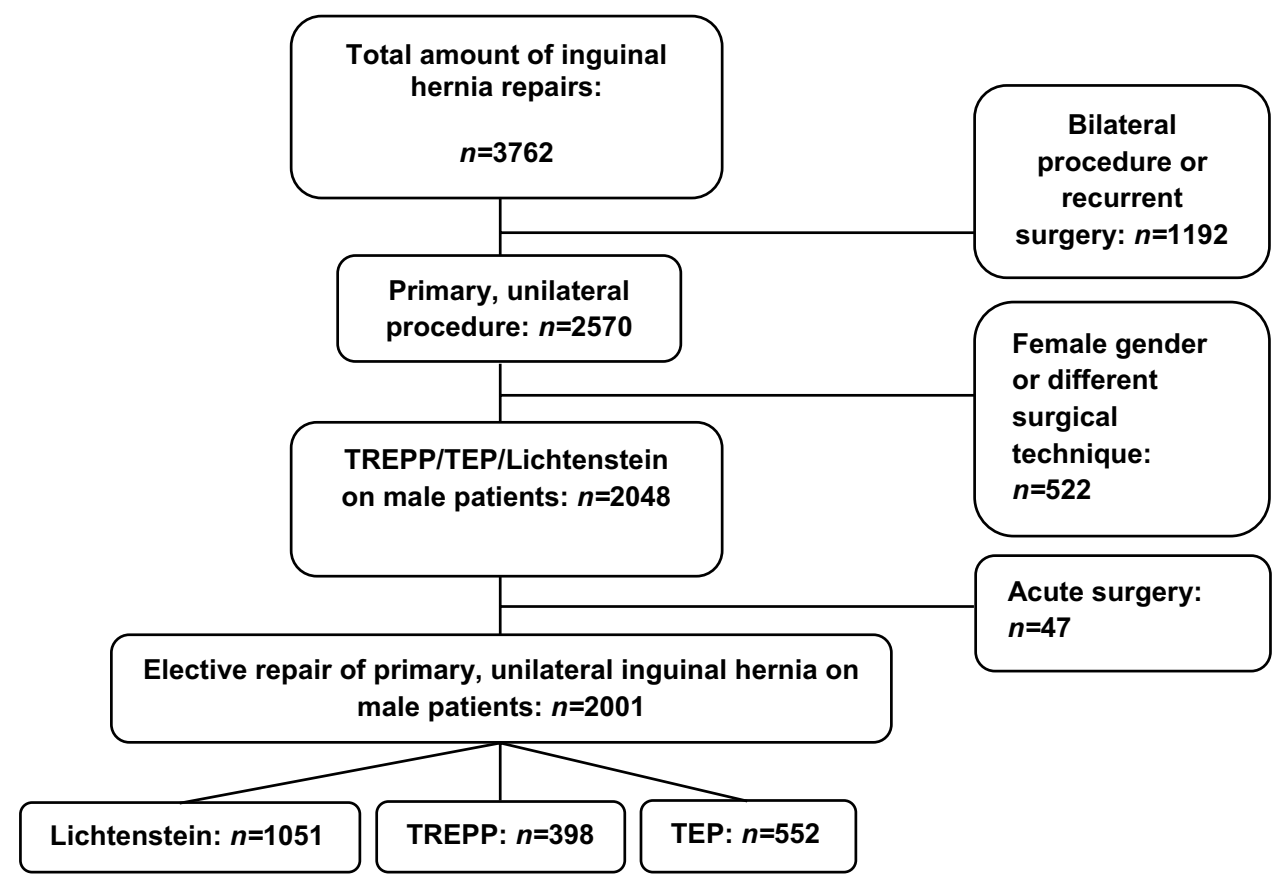


Table 1 Patient characteristics

\begin{tabular}{lccc}
\hline & TREPP $(n=398)$ & TEP $(n=552)$ & $\begin{array}{c}\text { Lichtenstein } \\
(n=1051)\end{array}$ \\
\hline $\begin{array}{l}\text { Mean age in } \\
\text { years (SD) }\end{array}$ & $57(14.6)$ & $54(15.4)$ & $64(15.1)$ \\
ASA class (\%) & & & \\
I (\%) & $163(41)$ & $215(39)$ & $286(28)$ \\
II (\%) & $201(51)$ & $295(54)$ & $519(51)$ \\
III (\%) & $34(8)$ & $39(7)$ & $208(20)$ \\
IV (\%) & $0(0)$ & $2(0)$ & $14(1)$ \\
\hline
\end{tabular}

Table 2 Postoperative outcomes TREPP versus Lichtenstein before propensity-score matching

\begin{tabular}{llll}
\hline & TREPP $(n=398)$ & $\begin{array}{l}\text { Lichten- } \\
\text { stein } \\
(n=1051)\end{array}$ & $p$ value \\
\hline Postoperative pain, $n(\%)$ & $29(7.3)$ & $44(4.2)$ & 0.022 \\
$\begin{array}{l}\text { Recurrence, } n(\%) \\
\begin{array}{l}\text { Surgical Site Occurrence, } \\
n(\%)\end{array}\end{array}$ & $15(3.8)$ & $27(2.6)$ & 0.22 \\
\hline
\end{tabular}

\section{TREPP versus Lichtenstein}

The presence of postoperative pain in the Lichtenstein cohort (before PSM) was significantly lower than in the TREPP cohort (Lichtenstein 4.2\% versus TREPP 7.3\%; $p=0.022$ ). There was no significant difference in the number of recurrences in both cohorts (Lichtenstein 2.6\% versus TREPP $3.8 \% ; p=0.22)$ The cumulative number of other adverse outcomes (e.g., hematoma, seroma, infection, urinary tract infection) was significantly higher in the Lichtenstein cohort $(13.0 \%)$ as compared to the TREPP cohort $(9.1 \% ; p=0.037)$. These numbers are shown in Table 2.

When comparing TREPP with Lichtenstein after PSM, all patients undergoing the TREPP procedure $(n=398)$ could be paired with a patient undergoing the Lichtenstein procedure, which created two groups with a total of 796 patients. The mean age of the non-matched group was significantly higher than the mean age in the matched pair group (mean age 68 versus 57; $p<0.001)$. The ASA-classification was significantly higher in the non-matched group $(p<0.001)$. When comparing TREPP versus Lichtenstein after PSM, the patients undergoing the TREPP technique did not experience significantly more postoperative pain as compared to the Lichtenstein group (TREPP 7.3\% versus Lichtenstein $6.3 \% ; p=0.67)$. The percentage of recurrences in the TREPP cohort was $3.8 \%$, which did not differ significantly compared to the Lichtenstein cohort $(2.5 \% ; p=0.42)$. The total number of adverse outcomes did not significantly differ between the two groups (TREPP 9.1\% versus Lichtenstein
Table 3 Comparison in postoperative outcomes in Lichtenstein and TREPP after propensity-score-matched analysis

\begin{tabular}{llll}
\hline & TREPP $(n=398)$ & $\begin{array}{l}\text { Lichten- } \\
\text { stein } \\
(n=398)\end{array}$ & $p$ value \\
\hline Postoperative pain, $n(\%)$ & $29(7.3)$ & $25(6.3)$ & 0.67 \\
$\begin{array}{l}\text { Recurrence, } n(\%) \\
\text { Surgical site occurrence, }\end{array}$ & $15(3.8)$ & $10(2.5)$ & 0.42 \\
$n(\%)$ & $36(9.1)$ & $48(12.1)$ & 0.20 \\
\hline
\end{tabular}

Table 4 Postoperative outcomes TREPP versus TEP before propensity-score matching

\begin{tabular}{llll}
\hline & TREPP $(n=398)$ & TEP $(n=552)$ & $p$ value \\
\hline Postoperative pain, $n(\%)$ & $29(7.3)$ & $28(5.1)$ & 0.17 \\
Recurrence, $n(\%)$ & $15(3.8)$ & $14(2.5)$ & 0.34 \\
$\begin{array}{l}\text { Surgical site occurrence, } \\
\quad 3(\%)\end{array}$ & $36(9.1)$ & $59(10.7)$ & 0.44 \\
\hline
\end{tabular}

$12.1 \% ; p=0.20)$. The odds ratio after exact conditional logistical regression for postoperative pain was 1.2 (confidence interval (CI) $0.65-2.14 ; p=0.67$ ), for recurrence 1.5 (CI $0.63-3.73 ; p=0.4$ ), for combined number of other adverse outcomes 0.7 (CI $0.44-1.18 ; p=0.2$ ). These results are shown in Table 3.

\section{TEP versus TREPP}

Prevalence of postoperative pain in the TEP cohort did not differ significantly compared to the TREPP procedure (TEP $5.1 \%$ versus TREPP $7.3 \%, p=0.17)$ and the prevalence of recurrent inguinal hernia in the TEP cohort was $2.5 \%$ (no significant difference with the TREPP cohort, 3.8\%; $p=0.34$ ). The cumulative total number of other adverse outcomes did not differ significantly between the two groups (TEP $10.7 \%$ versus TREPP 9.1\%; $p=0.44$ ). The results of TREPP versus TEP before PSM are shown in Table 4.

When comparing TREPP to the TEP technique after propensity-score matching, 390 pairs could be matched on propensity scores. This created two groups of a total of 780 patients. The non-matched group that remained (162 TEP patients, 8 TREPP patients) was significantly younger than the matched group (51 years versus 57 years; $p<0.001$ ). Furthermore, there was no difference in ASA-classification between both groups $(p=0.29)$. When comparing the TREPP to the TEP after PSM, no significant difference was present (TREPP $7.4 \%$ versus TEP $4.1 \% ; p=0.064$ ) in the presence of postoperative pain. Recurrences occurred in $3.9 \%$ of TREPP procedures versus $2.8 \%$ recurrences after the TEP procedure $(p=0.55)$. The cumulative total number of other adverse outcomes did not differ significantly 
however, the TREPP could play an important role in the decision-making for inguinal hernia repair. As an example, TREPP could play a useful part in recurrent hernia repair after previous anterior approach. Until now, the standard concept for treatment of recurrence after anterior approach is a posterior approach, mostly leading to a TEP (or TAPP) procedure. If the results from this study are indeed an accurate representation of the outcome, the TREPP procedure could take part in this flow chart, with possible advantages such as: single-incision access viable for intra-peritoneal inspection, pre-peritoneal location of mesh, possibility of regional anesthesia and potential lower costs due to the fact that laparoscopic equipment is not needed.

Author contributions TZ design of study, preparation, database creation, analysis of data and co-writing paper. NS database creation and co-writing paper. NV analysis of data and co-writing paper. MM study design (institutional board procedures), essential data delivery, cowriting of paper. DH study design (institutional board procedures), essential data delivery, co-writing of paper. EJ study design, database creation, co-writing of paper. HB study design (institutional board procedures), essential data delivery, co-writing of paper. PV providing essential data for database, co-writing paper, supervising the research. GK design of study, co-writing paper, supervising the research. HE design of study, analysis of data, supervising the research and cowriting. JP design of study, analysis of data, supervising the research and co-writing.

Funding Not applicable.

Availability of data and material Is available on request.

\section{Compliance with ethical standards}

Conflict of interest None of the authors have conflicts of interests.

Ethical approval The Medical Ethics Committee of our institution (RTPO Leeuwarden) confirmed the conduct of this retrospective study without the need for ethical review, and the institutional boards of the four hospitals approved the execution of the study without the need for consent in accordance with Dutch regulations.

Human and animal rights The procedures performed in this study are approved procedures for daily-practice, and this descriptive retrospective study therfore does not includeexperiments on humans.

Informed consent Not applicable.

Code availability Access via a formal application process.

Open Access This article is licensed under a Creative Commons Attribution 4.0 International License, which permits use, sharing, adaptation, distribution and reproduction in any medium or format, as long as you give appropriate credit to the original author(s) and the source, provide a link to the Creative Commons licence, and indicate if changes were made. The images or other third party material in this article are included in the article's Creative Commons licence, unless indicated otherwise in a credit line to the material. If material is not included in the article's Creative Commons licence and your intended use is not permitted by statutory regulation or exceeds the permitted use, you will need to obtain permission directly from the copyright holder. To view a copy of this licence, visit http://creativecommons.org/licenses/by/4.0/.

\section{References}

1. HerniaSurge Group (2018) International guidelines for groin hernia management. Hernia 22:1-165

2. Statline, Centraal Bureau voor de Statistiek - https://opendata.cbs. nl/statline/\#/CBS/nl/dataset/80386ned/table?ts=1555317940875, (2014). Accessed 12 Apr 2019

3. Fitzgibbons RJ Jr, Giobbie-Hurder A, Gibbs JO, Dunlop DD, Reda DJ, McCarthy M Jr et al (2006) Watchful waiting vs repair of inguinal hernia in minimally symptomatic men: a randomized clinical trial. JAMA 295:285-292

4. Schroeder AD, Tubre DJ, Fitzgibbons RJ Jr (2019) Watchful waiting for inguinal hernia. Adv Surg 53:293-303

5. Langeveld HR, van't Riet M, Weidema WF, Stassen LP, Steyerberg EW, Lange $\mathbf{J}$ et al (2010) Total extraperitoneal inguinal hernia repair compared with Lichtenstein (the LEVEL-Trial): a randomized controlled trial. Ann Surg 251:819-824

6. Classification of chronic pain (1986) Descriptions of chronic pain syndromes and definitions of pain terms. Prepared by the International Association for the Study of Pain, Subcommittee on Taxonomy. Pain Suppl 3:S1-S226

7. Nienhuijs S, Staal E, Strobbe L, Rosman C, Groenewoud H, Bleichrodt $R$ (2007) Chronic pain after mesh repair of inguinal hernia: a systematic review. Am J Surg 194:394-400

8. Eklund A, Montgomery A, Bergkvist L, Rudberg C, Swedish Multicentre Trial of Inguinal Hernia Repair by Laparoscopy (SMIL) study group (2010) Chronic pain 5 years after randomized comparison of laparoscopic and Lichtenstein inguinal hernia repair. Br J Surg 97:600-608

9. Koning GG, Keus F, Koeslag L, Cheung CL, Avci M, van Laarhoven CJ et al (2012) Randomized clinical trial of chronic pain after the transinguinal preperitoneal technique compared with Lichtenstein's method for inguinal hernia repair. Br J Surg 99:1365-1373

10. Neumayer L, Giobbie-Hurder A, Jonasson O, Fitzgibbons R Jr, Dunlop D, Gibbs J et al (2004) Open mesh versus laparoscopic mesh repair of inguinal hernia. N Engl J Med. 350:1819-1827

11. Lange JF, Lange MM, Voropai DA, van Tilburg MW, Pierie JP, Ploeg RJ et al (2014) Trans rectus sheath extra-peritoneal procedure (TREPP) for inguinal hernia: the first 1000 patients. World J Surg 38:1922-1928

12. Kockerling $\mathrm{F}$ (2019) TEP for elective primary unilateral inguinal hernia repair in men: what do we know? Hernia 23:439-459

13. Koning GG, Andeweg CS, Keus F, van Tilburg MW, van Laarhoven CJ, Akkersdijk WL (2012) The transrectus sheath preperitoneal mesh repair for inguinal hernia: technique, rationale, and results of the first 50 cases. Hernia 16:295-299

Publisher's Note Springer Nature remains neutral with regard to jurisdictional claims in published maps and institutional affiliations. 